DOI: $10.2478 /$ bjmg-2014-0071

ORIGINAL ARTICLE

\title{
INTELLECTUAL ABILITY IN THE DUCHENNE MUSCULAR DYSTROPHY AND DYSTROPHIN GENE MUTATION LOCATION
}

Milic Rasic $\mathrm{V}^{1,2, *}$, Vojinovic $\mathrm{D}^{1}$, Pesovic $\mathrm{J}^{3}$, Mijalkovic $\mathrm{G}^{1}$, Lukic $\mathrm{V}^{1}$, Mladenovic $\mathrm{J}^{1}$, Kosac A ${ }^{1}$, Novakovic I ${ }^{4}$, Maksimovic $\mathrm{N}^{4}$, Romac $\mathrm{S}^{3}$, Todorovic $\mathrm{S}^{1}$, Savic Pavicevic $\mathrm{D}^{3}$

\begin{abstract}
*Corresponding Author: Vedrana Milic Rasic, M.D., Ph.D., Clinic for Neurology and Psychiatry for Children and Youth, Dr. Subotica 6A, 11000 Belgrade, Serbia. Tel: +381-11-265-8355. Fax: +381-11-264-5064. E-mail: vedrana.milic.npk@gmail.com
\end{abstract}

\begin{abstract}
Duchenne muscular dystrophy (DMD) is the most common form of muscular dystrophy during childhood. Mutations in dystrophin $(D M D)$ gene are also recognized as a cause of cognitive impairment. We aimed to determine the association between intelligence level and mutation location in $D M D$ genes in Serbian patients with DMD. Forty-one male patients with DMD, aged 3 to 16 years, were recruited at the Clinic for Neurology and Psychiatry for Children and Youth in Belgrade, Serbia. All patients had defined $D M D$ gene deletions or duplications [multiplex ligation-dependent probe amplification (MLPA), polymerase chain reaction (PCR)] and cognitive status assessment (Wechsler Intelligence Scale for Children, Brunet-Lezine scale, Vineland-Doll scale). In 37 patients with an estimated full scale intelligence quotient (FSIQ), six (16.22\%) had borderline intelligence $(70<$ FSIQ $\leq 85)$, while seven $(18.92 \%)$ were intellectually impaired (FSIQ <70). The FSIQ was not associated with proximal and distal mutations when boundaries were set at exons 30 and 45. However, FSIQ was statistically significantly associated with mutation location when we assumed their func-

\footnotetext{
${ }^{1}$ Clinic for Neurology and Psychiatry for Children and Youth, Belgrade, Serbia

2 Faculty of Medicine, University of Belgrade, Belgrade, Serbia

${ }^{3}$ Center for Human Molecular Genetics, Faculty of Biology, University of Belgrade, Belgrade, Serbia

${ }^{4}$ Institute of Human Genetics, Faculty of Medicine, University of Belgrade, Belgrade, Serbia
}

tional consequence on dystrophin isoforms and when mutations in the 5'-untranslated region (5'UTR) of Dp140 (exons 45-50) were assigned to affect only Dp427 and Dp260. Mutations affecting Dp140 and Dp71/Dp40 have been associated with more frequent and more severe cognitive impairment. Finally, the same classification of mutations explained the greater proportion of FSIQ variability associated with cumulative loss of dystrophin isoforms. In conclusion, cumulative loss of dystrophin isoforms increases the risk of intellectual impairment in DMD and characterizing the genotype can define necessity of early cognitive interventions in DMD patients.

Keywords: Duchenne muscular dystrophy (DMD); dystrophin isoform; intellectual impairment.

\section{INTRODUCTION}

Duchenne muscular dystrophy (DMD) is the most common form of muscular dystrophy during childhood, affecting 1 in 3500 live born males [1]. This fatal, $\mathrm{X}$ linked disease, leads to progressive muscular weakness and less well described non progressive central nervous system manifestations.

The consistent finding in patients with DMD is reduction in a full-scale intelligence quotient (FSIQ) by one standard deviation (SD) from the population mean $[1,2]$. Although most affected boys are not intellectually disabled, the risk of cognitive impairment is increased in DMD patients. Therefore, up to $30.0 \%$ of patients have intellectual disability with a FSIQ of less than 70 , including around $3.0 \%$ of them with 
severe impairment and FSIQ of less than $50[2,3]$. Duchenne muscular dystrophy is caused by various types of mutations within the dystrophin gene $(D M D)$ [4], which changes the reading frame of coding transcripts affecting the production of protein dystrophin [5]. Expression of the dystrophin is controlled by three upstream promoters, which produce full-length dystrophin isoform (Dp427) and four internal promoters that regulate production of shorter dystrophin isoforms (Dp260, Dp140, Dp116 and Dp71) [6-9]. Dp427 is expressed in skeletal and cardiac muscle, brain and Purkinje cells, Dp260 is expressed in retina [10], Dp140 in brain, retina and kidney [11], while Dp116 is present in peripheral nerves [12]. Dp71 is the most abundant isoform in non muscular tissues and represents the major product in the adult brain [13]. The dystrophin is a part of dystrophinassociated glycoprotein complex, and in the brain, it is involved in the clustering of ion channels and post synaptic membrane receptors during synaptogenesis [9], suggesting that loss of its function may be responsible for intellectual impairment and cognitive deficits in DMD patients. Cognitive deficit is likely the result of cumulative loss of Dp427, Dp140 and Dp71 [9,14], whereas loss of Dp71 contributes to the severity of cognitive impairment [14,15]. Recently, one more dystrophin isoform (Dp40), produced from the same promoter as Dp71 but by the use of an alternative polyadenylation site, has been implicated in pre synaptic function [16].

Although cognitive impairment has frequently been reported, systematized data on the cognitive profile of patients with DMD in Serbia is lacking. Therefore, the aim of this study was to determine frequency of intellectual impairment and to examine association of intelligence level with mutation location and affected dystrophin isoforms among our patients with DMD.

\section{MATERIALS AND METHODS}

Patient Data. Forty-one patients with DMD were recruited retrospectively at the Clinic for Neurology and Psychiatry for Children and Youth in Belgrade, Serbia, during the period between 1992 and 2013. The diagnosis of DMD was based on the clinical onset of the disease before 5 years of age, initial or clear neurological signs of decline of motor function at the age of 6 years, decline of motor function or positive family history of DMD for boys younger than 6 years, elevated serum creatine kinase levels and confirmed mutation in the DMD gene. All recruited patients were unrelated except of one sibling pair.

All patients and/or their parents gave informed consent concerning the use of the data for research. This study was approved by the Ethics Committee of the Clinic for Neurology and Psychiatry for Children and Youth, Belgrade, Serbia.

Methods: Genetic Analysis. Deletions and duplications were detected via multiplex ligationdependent probe amplification (MLPA) using two probe mixes, P034 and P035 (MRC Holland, Amsterdam, The Netherlands) [17] at the Center for Human Molecular Genetics at the Faculty of Biology in Belgrade, Serbia. A few samples were analyzed via multiplex polymerase chain reaction (PCR) [18-20] at the Institute of Human Genetics at the Faculty of Medicine in Belgrade, Serbia, were included in the analyses as the mutation location allowed us to unequivocally assign the altered dystrophin isoforms.

Mutations were described using Human Genome Variation Society (HGVS) nomenclature [21]. The positions of the mutations were determined in relation to reference sequence NM_004006 (GenBank) at cDNA level and in relation to reference sequence UniProtKB:11532 (Uni ProtKB/Swiss-Prot) at protein level. The mutation effect on the reading frame was determined using software $D M D$ gene reading frame checker (available at http://www. dmd.nl/).

Since the effect of mutation location on FSIQ was expected to be different, all mutations were divided into two structural groups according to previously applied classifications $[22,23]$. The mutations localized upstream from exon $30(1-30)$ and the mutations localized upstream from exon 45 (1-45) were defined as proximal mutations, while the mutations downstream from exon 30 (31-79) or exon 45 (46-79) were considered as distal mutations.

Considering the complex organization of $D M D$ gene and dystrophin isoforms produced from the inner promoters, mutations were assigned to altered expression of dystrophin isoforms (Dp427, Dp260, Dp140, Dp116, Dp71 and Dp40), and were divided into various groups. We took into consideration that the Dp140 transcript has a long 5' untranslated region (5'UTR), consisting of exons 45-50, so that the effect of mutations in this region on Dp140 expression 
could not be confidently predicted. Therefore, we also analyzed clustering, in which mutations within this region (Dp140utr) were assumed to be coding exon mutations affecting only expression of Dp427 and Dp260 but not Dp140, while mutations in the promoter and protein coding region of Dp140 (Dp140pc) were assigned to affect expression of Dp140 [14]. As the promoter that regulates Dp140 expression lies within intron 44 of the $D M D$ gene, all patients who had a deletion breakpoint within intron 44 were tested by PCR for the presence of the Dp140 promoter using the following primers: IN44F (5'-GCC CTA AGT GCT TCC AGA AA-3') and IN44R (5'-CTC ACA GCT CCT GCA TCA GA-3'). This original approach allowed us to accurately group patients with the affected Dp140 expression. To assess the cumulative effect of dystrophin isoforms on FSIQ, the patients were divided into three groups with respect to the preservation or loss of Dp140 and Dp71/Dp40.

Cognitive Assessment. All DMD patients were psychologically tested. Taking into account the patients' age, different psychological instruments were used for cognitive status assessment.

In order to assess intelligence level in children younger than 16 years and 11 months, the Wechsler Intelligence Scale for Children (WISC) was used [24]. The test generates FSIQ, which represents overall cognitive ability. Patients with FSIQ $\leq 70$ were considered mentally disabled, while patients with FSIQ ranging between 70 and $85(70<$ FSIQ $\leq 85)$ were defined as borderline.

The Brunet-Lezine scale, statistically adapted for our population, was applied in order to assess psychomotor developmental quotient (DQ) in children from 0 to 30 months [25]. With complementary tests, this scale can be used until 6 years of age.

The Vineland-Doll scale was used to measure social maturity in individuals with mental retardation or individuals who had difficulty performing in testing situations [26]. Based on literature findings, an estimate of social quotient (SQ) provided by this test, was highly correlated (0.80) with intelligence.

Statistical Analysis. Exploration of normality, assessed with the Shapiro-Wilk test, revealed normal distribution of age and FSIQ in our sample. Therefore, parametric tests were used for further analysis. To investigate whether the mean FSIQ was statistically different from normative values $(100 \pm 15)$ one sample t-test was applied. To evaluate whether age had an impact on cognitive status, the Pearson correlation coefficient was calculated. The results were considered significant when probability was less than 0.05 .

The cognitive abilities of patients with mutations localized in proximal and distal parts of the $D M D$ gene were compared using the parametric one-way $t$-test for independent samples. The same test was used to assess an effect of loss of different dystrophin isoforms on FSIQ. In order to adjust for multiple testing, we made an adjustment of $p$ value calculating Bonfferoni correction $(0.05 / 3$ tests $=0.017)$. To determine whether Dp140 and Dp71/Dp40 were associated with cognitive abilities, patients with presumably intact or absent Dp140 and/or Dp71/Dp40 were compared using oneway ANOVA analysis of variance. Statistical analyses were performed using commercially available software (Statistical Package for the Social Sciences, IBM Corporation, Armonk, NY, USA; Statistics for Windows, SPSS Version 20.0).

\section{RESULTS}

Clinical and Genetic Characteristics. Fortyone patients with genetically confirmed deletion or duplication in the DMD gene and cognitive status assessment were recruited at the Clinic for Neurology and Psychiatry for Children and Youth in Belgrade, Serbia. All affected patients were males, aged 3 to 16 $(8.34 \pm 2.56)$ years. Deletions were confirmed in 37 patients $(90.24 \%)$, while duplications were identified in four patients $(9.75 \%)$. The identified deletions and duplications, description of the mutations at cDNA and protein level, mutation effect on reading frame, as well as dystrophin isoforms impaired by mutation, the age at the onset of disease, the age at psychological testing and data of the psychological exploration are shown in Table 1. Although patients with in-frame mutations could express milder phenotypes, all our patients with in-frame mutations developed a DMD phenotype: one patient showed delayed psychomotor development and was wheelchair-bound at the age of 10 (ID 28), the second had the onset of the disease at the age of 3.5 years and developed positive Gowers sign at the age of 7 (ID 5), and two boys, the youngest one (IDs 2 and 38), expressed initial signs of motor decline at the age of 6 .

The FSIQ was estimated for 37 participants, DQ was estimated for two patients, while SQ was as- 
Table 1. Observed deletions and duplications for all examined patients with Duchenne muscular dystrophy and corresponding cognitive abilities.

\begin{tabular}{|c|c|c|c|c|c|c|c|c|}
\hline ID & $\begin{array}{c}\text { Age at } \\
\text { Onset } \\
\text { (years) }\end{array}$ & $\begin{array}{l}\text { Detected } \\
\text { DMD Gene }\end{array}$ & $\begin{array}{l}\text { Mutation at } \\
\text { cDNA Level }\end{array}$ & $\begin{array}{l}\text { Mutation at } \\
\text { Protein Level }\end{array}$ & $\begin{array}{l}\text { Mutation } \\
\text { Effect }\end{array}$ & $\begin{array}{l}\text { Affected Dystrophin } \\
\text { Isoforms }\end{array}$ & FSIQ & $\begin{array}{c}\text { Age at } \\
\text { FSIQ } \\
\text { Test } \\
\text { (years) }\end{array}$ \\
\hline 1 & 5 & $\begin{array}{l}\text { deletion of exon } 1 \text { and } \\
\text { Dp } 427 \mathrm{c}\end{array}$ & c.(?_-128297)_31+?del & N/A & $\mathrm{N} / \mathrm{A}$ & Dp427 & 72 & 9 \\
\hline 2 & 5 & deletion of exons 3_4 & c.94-?_264+?del & p.F32_N88del & in frame & Dp427 & 100 & 6 \\
\hline 3 & 3 & duplication of exons $3 \_7$ & c.94-?_649+?dup & p.D217V fs*7 & out of frame & Dp427 & 96 & 11 \\
\hline 4 & 2 & deletion of exons $3 \_17$ & c.94-?_2168+?del & p.L724V fs*4 & out of frame & Dp427 & 75 & 9 \\
\hline 5 & 3.5 & deletion of exons $3 \_18$ & c.94-?_2292+?del & p.F32_N764del & in frame & Dp427 & 106 & 7 \\
\hline 6 & 2 & duplication of exons $8 \_12$ & c.650-?_1482+?dup & p.V495M fs*13 & out of frame & Dp427 & 110 & 10 \\
\hline 7 & 2 & duplication of exons 16_17 & c.1813-?_2168+?dup & p.L724F fs*2 & out of frame & Dp427 & 88 & 10 \\
\hline 8 & 5 & deletion of exons 20_23 & c.2381-?_3162+?del & p.N1055E fs* 12 & out of frame & Dp427 & 111 & 8 \\
\hline 9 & 3 & deletion of exons 8_34 & c.650-?_4845+?del & p.A1616G fs*7 & out of frame & Dp427, Dp260 & 100 & 6 \\
\hline 10 & 2 & deletion of exon $45^{\mathrm{a}}$ & c.6439-?_6614+?del & p.L2206A fs*17 & out of frame & Dp427, Dp260, Dp140utr & 109 & 9 \\
\hline 11 & 2 & deletion of exons $45 \_50^{\mathrm{a}}$ & c.6439-?_7309+?del & p.S2437L fs*9 & out of frame & Dp427, Dp260, Dp140utr & 96 & 5 \\
\hline 12 & 5 & deletion of exons $45 \_50^{\mathrm{a}}$ & c.6439-?_7309+?del & p.S2437L fs*9 & out of frame & Dp427, Dp260, Dp140utr & 118 & 9 \\
\hline 13 & 2 & deletion of exons $46 \_47$ & c.6615-?_6912+?del & p.V2305F fs*16 & out of frame & Dp427, Dp260, Dp140utr & 105 & 10 \\
\hline 14 & 4 & deletion of exons $46 \_48$ & c.6615-?_7098+?del & p.E2367K fs*4 & out of frame & Dp427, Dp260, Dp140utr & 68 & 6 \\
\hline 15 & pdd & deletion of exons 46_50 & c.6615-?_7309+?del & p.R2205S fs*16 & out of frame & Dp427, Dp260, Dp140utr & 94 & 7 \\
\hline 16 & $<2$ & deletion of exons 46_50 & c.6615-?_7309+?del & p.R2205S fs*16 & out of frame & Dp427, Dp260, Dp140utr & 114 & 9 \\
\hline 17 & 3 & deletion of exons 48 _50 & c.6913-?_7309+?del & p.S2437L fs*9 & out of frame & Dp427, Dp260, Dp140utr & 89 & 10 \\
\hline 18 & $<2$ & deletion of exons 48 _50 & c.6913-?_7309+?del & p.S2437L fs*9 & out of frame & Dp427, Dp260, Dp140utr & 94 & 7 \\
\hline 19 & 4 & deletion of exons 49_50 & c.7099-?_7309+?del & p.S2437L fs*9 & out of frame & Dp427, Dp260, Dp140utr & 63 & 7 \\
\hline 20 & 5 & deletion of exons 49_50 & c.7099-?_7309+?del & p.S2437L fs*9 & out of frame & Dp427, Dp260, Dp140utr & 99 & 9 \\
\hline 21 & $<2$ & deletion of exon 50 & c.7201-?_7309+?del & p.S2437L fs*9 & out of frame & Dp427, Dp260, Dp140utr & 63 & 12 \\
\hline 22 & pdd & deletion of exon 50 & c.7201-?_7309+?del & p.S2437L fs*9 & out of frame & Dp427, Dp260, Dp140utr & 95 & 6 \\
\hline 23 & 4.5 & deletion of exon $44^{\mathrm{a}}$ & c.6291-?_6438+?del & p.E2147N fs*16 & out of frame & Dp427, Dp260, Dp140pc & 71 & 8 \\
\hline 24 & 4 & deletion of exon $44^{a}$ & c.6291-?_6438+?del & p.E2147N fs*16 & out of frame & Dp427, Dp260, Dp140pc & 94 & 11 \\
\hline 25 & 3.5 & deletion of exons $452^{52^{\mathrm{a}}}$ & c.6439-?_7660+?del & p.I2554L fs*22 & out of frame & Dp427, Dp260, Dp140pc & 50 & 9 \\
\hline 26 & 4 & deletion of exons 46_51 & c.6615-?_7542+?del & p.A2515Q fs*23 & out of frame & Dp427, Dp260, Dp140pc & 95 & 14 \\
\hline 27 & 2.5 & deletion of exons 46_52 & c.6615-?_7660+?del & p.R2205S fs*2 & out of frame & Dp427, Dp260, Dp140pc & 88 & 9 \\
\hline 28 & pdd & deletion of exons 47_51 & c.6763-?.7542+?del & p.L2255_K2514del & in frame & Dp427, Dp260, Dp140pc & 96 & 10 \\
\hline 29 & N/A & deletion of exons 50_5 $3^{\mathrm{b}}$ & N/A & N/A & N/A & Dp427, Dp260, Dp140pc & 75 & 7 \\
\hline 30 & 5 & deletion of exons $50 \_53^{b}$ & N/A & N/A & N/A & Dp427, Dp260, Dp140pc & 79 & 7 \\
\hline 31 & 3.5 & deletion of exon 51 & c.7310-?_7542+?del & p.A2515C fs $* 33$ & out of frame & Dp427, Dp260, Dp140pc & 87 & 11 \\
\hline 32 & 5 & deletion of exon 52 & c.7543-?_7660+?del & p.I2554L fs $* 22$ & out of frame & Dp427, Dp260, Dp140pc & 101 & 11 \\
\hline 33 & 3 & deletion of exon $53^{b}$ & N/A & N/A & N/A & Dp427, Dp260, Dp140pc & 98 & 7 \\
\hline
\end{tabular}


BALKAN JOURNAL OF MEDICAL GENETICS

Milic Rasic V, Vojinovic D, Pesovic J, Mijalkovic G, Lukic V, Mladenovic J, Kosac A, Novakovic I, Maksimovic N, Romac S, Todorovic S, Savic Pavicevic D

\begin{tabular}{|c|c|l|l|l|l|l|c|c|}
\hline 34 & $<2$ & deletion of exon 53 & c.7661-?_7872+?del & p.Q2625T fs*18 & out of frame & Dp427, Dp260, Dp140pc & 84 & 6 \\
\hline 35 & 4 & deletion of exon 61 & c.9084-?_9163+?del & p.T3055R fs*34 & out of frame & $\begin{array}{l}\text { Dp427, Dp260, Dp140pc, } \\
\text { Dp116 }\end{array}$ & 55 & 8 \\
\hline 36 & 2.5 & deletion of exons 45_73a & c.6439-?_10394+?del & p.D3466R fs*2 & out of frame & $\begin{array}{l}\text { Dp427, Dp260, Dp140pc, } \\
\text { Dp116, Dp71, Dp40 }\end{array}$ & 44 & 16 \\
\hline 37 & pdd & deletion of exons 45_76a & c.6439-?_10921+?del & p.G3641V fs*16 & out of frame & $\begin{array}{l}\text { Dp427, Dp260, Dp140pc, } \\
\text { Dp116, Dp71, Dp40 }\end{array}$ & 58 & 7 \\
\hline 38 & 5 & deletion of exons 10_23 & c.961-?_3162+?del & p.H321_Q1054del & in frame & Dp427 & SQ 41 & 6 \\
\hline 39 & 3 & deletion of exons 48_50 & c.6913-?_7309+?del & p.S2437L fs*9 & out of frame & Dp427, Dp260, Dp140utr & DQ 105 & 3 \\
\hline 40 & 4 & deletion of exons 45_76a & c.6439-?_10921+?del & p.G3641V fs*16 & out of frame & $\begin{array}{l}\text { Dp427, Dp260, Dp140pc, } \\
\text { Dp116, Dp71, Dp40 }\end{array}$ & DQ 82 & 4 \\
\hline 41 & pdd & $\begin{array}{l}\text { duplication of exons 52_55, } \\
\text { duplication of exons 63_67, } \\
\text { triplication of exons 68_79 }\end{array}$ & $\begin{array}{l}\text { c.7543-?_8225-?_9807+?dup, } \\
\text { c.9808-?_(*2691_?)trip }\end{array}$ & p.A3270P fs*22 & out of frame & $\begin{array}{l}\text { Dp427, Dp260, Dp140pc, } \\
\text { Dp116, Dp71, Dp40 }\end{array}$ & SQ 58 & 6 \\
\hline
\end{tabular}

ID: identification number; FSIQ: full scale intelligence quotient; N/A: not available; ppd: psychomotor development delay; SQ: social quotient; DQ: developmental quotient. Mutations at the cDNA and protein levels were described according to nomenclature suggested by the Human Genome Variation Society (HGVS), (15); mutations at the cDNA level were determined in relation to the reference sequence NM 004006 (GenBank), and at the protein level in relation to reference sequence UniProtKB:P11532 (UniProtKB/Swiss-Prot); mutation effect on reading frame was determined using software $D M D$ gene reading frame checker (available at http://www.dmd.nl/).

a The patient was tested for the presence of the Dp140 promoter region in intron 44.

${ }^{\mathrm{b}}$ The mutation was detected with multiplex PCR; therefore, it was not possible to identify its precise nomenclature on cDNA and protein levels, or its effect on the open reading frame.

sessed for two patients. The patients with estimated DQ and SQ were excluded from statistical analysis and they are described separately (Table 2).

General intelligence evaluation of the analyzed population demonstrated statistically significant difference from population normative values $(t=$ $-4.024, p=0.00015)$. The FSIQ with mean of 87.57 (SD 18.79) had a broad range of values between 44.0 and 118.0. Thirteen patients (35.14\%) had FSIQ lower than 85 , of whom six patients $(16.22 \%)$ had borderline intelligence levels $(70<\mathrm{FSIQ} \leq 85)$, while seven patients $(18.92 \%)$ were considered intellectu-

Table 2. Scores according to different psychological tests.

\begin{tabular}{|l|c|c|}
\hline $\begin{array}{l}\text { Psychological } \\
\text { Exploration }\end{array}$ & $\boldsymbol{n}$ & $\begin{array}{c}\text { Mean Value } \\
\text { (range) }\end{array}$ \\
\hline FSIQ & 37 & $\begin{array}{c}(87.57 \pm 18.79) \\
(44,118)\end{array}$ \\
\hline DQ & 2 & $(82,105)$ \\
\hline SQ & 2 & $(41,58)$ \\
\hline
\end{tabular}

$n$ : number of patients; FSIQ: full scale intelligence quotient; DQ: development quotient; SQ: social quotient. ally disabled (FSIQ $\leq 70)$. Two intellectually disabled patients were severely impaired (FSIQ $<50)$. Above average FSIQ was assessed in four patients (FSIQ $\geq 110$ ). Assessed correlation coefficient showed no significant association between age and FSIQ (Pearson's $r=-0.129$ ).

Association of Intelligence Level with Mutation Location and Affected Dystrophin Isoforms. The analysis of the intellectual ability with respect to the structural classification of mutation locations did not show statistically significant difference in the mean FSIQ between the patients with mutation proximal and distal to exon $30(t=1.23, p=0.114)$ (Table $3)$. The patients with mutation proximal to exon 30 had a mean FSIQ of 94.75 (SD 15.14), while for the patients with mutations distal to exon 30 , the mean FSIQ was 88.59 (SD 19.44). A similar result was observed when exon 45 was used as a boundary $(t=1.45$, $p=0.130$ ) (Table 3 ). The patients with the mutation proximal to exon 45 had a mean FSIQ of 93.0 (SD 14.71), while for the patients with mutation in the distal part, the mean FSIQ was 85.27 (SD 20.09).

However, the analysis of intellectual ability with respect to mutation location assigned to the impaired 
Table 3. Association between proximal and distal mutations and full scale intelligence quotient.

\begin{tabular}{|c|c|c|c|}
\hline Location of Mutation in the DMD Gene & $n$ & Mean FSIQ (SD) & $t$-Test \\
\hline $\begin{array}{l}\text { Proximal to exon } 30(1-30) \\
\text { Distal to exon } 30(31-79)\end{array}$ & $\begin{array}{r}8 \\
29\end{array}$ & $\begin{array}{l}94.75(15.14) \\
88.59(19.44)\end{array}$ & $t=1.23 ; d f=35 ; p=0.114$ \\
\hline $\begin{array}{l}\text { Proximal to exon } 45(1-45) \\
\text { Distal to exon } 45(46-79)\end{array}$ & $\begin{array}{l}11 \\
26\end{array}$ & $\begin{array}{l}93.00(14.71) \\
85.27(20.09)\end{array}$ & $t=1.45 ; d f=35 ; p=0.130$ \\
\hline
\end{tabular}

FSIQ: full scale intelligence quotient; $n$ : number of patients; SD: standard deviation.

dystrophin isoforms indicated statistically significantly different scoring between patients whose mutations affected expression of Dp427 and Dp260, and patients whose mutations additionally affected expression of Dp140, Dp116, Dp71 and Dp40 $(t=2.67$, $p=0.0057$ ) (Table 4). This result was obtained after the group of patients with a mutation in the Dp140utr region was clustered together with the patients whose mutations altered expression of Dp427 and Dp260. The frequency of borderline intellectual ability in the group of patients with altered Dp427, Dp260 and Dp140utr, was $9.09 \%$, while in the group of patients with altered Dp140pc, Dp116, Dp71 and Dp40, it was $26.67 \%$. The frequency of intellectual disability in the two groups was 13.64 vs. $26.67 \%$. Furthermore, three patients had affected Dp116, Dp71 and Dp40 and they were intellectually disabled (IDs 36, 37 and 41). Considering the few observations in this group of patients, a statistical test was not performed.

Additionally, 28 patients with the mutation affecting Dp140, were classified into groups based on the mutation localization in Dp140utr or Dp140pc. The patients with mutation within the Dp140utr region had a mean FSIQ of 92.85 (SD 18.16), while for patients with a mutation in the Dp140pc region, the mean FSIQ was 78.33 (SD 18.79). The difference in the mean FSIQ was statistically significantly different $(t=2.07, p=0.024)$ between those two groups.

Finally, the distribution of FSIQ with respect to functional consequence on the Dp140 and Dp71/ Dp40 isoforms indicated that cognitive impairment in DMD patients was associated with the cumulative loss of dystrophin isoforms (Figure 1). A greater proportion of variability in FSIQ was explained after assuming that mutations within the Dp140utr affect the functional loss of Dp427 and Dp260, but not $\operatorname{Dp} 140(F=7.454, p=0.002$ vs. $F=5.76, p=0.007)$.

For the youngest two patients, psychomotor development was measured with a scale for early infancy. Estimated DQ suggested normal psychomotor development ( $\mathrm{DQ}=105$ ) for a boy (ID 39) whose mutation affected expression of Dp427, Dp260 and

Table 4. Association between mutation locations assigned to altered expression of dystrophin isoforms and full scale intelligence quotient.

\begin{tabular}{|c|c|c|c|c|}
\hline Location of the Mutation in the DMD Gene & Affected Dystrophin Isoforms & $n$ & Mean FSIQ (SD) & $t$-Test \\
\hline $\begin{array}{l}\text { Proximal to intron } 29 \\
\text { Distal to intron } 29\end{array}$ & $\begin{array}{l}\text { Dp427 } \\
+ \text { Dp260, Dp140, Dp116, Dp71, Dp40 }\end{array}$ & $\begin{array}{r}8 \\
29\end{array}$ & $\begin{array}{l}94.75(15.14) \\
85.59(19.44)\end{array}$ & $t=1.23, d f=35, p=0.113$ \\
\hline $\begin{array}{l}\text { Proximal to intron } 44 \\
\text { Dp140 promoter and distal to intron } 44\end{array}$ & $\begin{array}{l}\text { Dp427, Dp260 } \\
\text { +Dp140, Dp116, Dp71, Dp40 }\end{array}$ & $\begin{array}{r}9 \\
28\end{array}$ & $\begin{array}{l}95.33(14.27) \\
85.07(19.59)\end{array}$ & $t=1.45, d f=35, p=0.078$ \\
\hline $\begin{array}{l}\text { Proximal to exon } 51 \text { excluding the Dp } 140 \text { promoter } \\
\text { Dp140 promoter and distal to exon } 51 \text { including exon } 51\end{array}$ & $\begin{array}{l}\text { Dp427, Dp260, Dp140utr } \\
\text { +Dp140pc, Dp116, Dp71, Dp40 }\end{array}$ & $\begin{array}{l}22 \\
15\end{array}$ & $\begin{array}{l}93.86(16.36) \\
78.33(18.79)\end{array}$ & $t=2.67, d f=35, \boldsymbol{p}=\mathbf{0 . 0 0 5 7} 7^{\mathrm{a}}$ \\
\hline $\begin{array}{l}\text { Proximal to intron } 55 \\
\text { Distal to intron } 55\end{array}$ & $\begin{array}{l}\text { Dp427, Dp260, Dp140 } \\
+ \text { +Dp116, Dp71, Dp40 }\end{array}$ & $\begin{array}{r}34 \\
3\end{array}$ & $\begin{array}{l}90.68(16.10) \\
52.33(7.37)^{b}\end{array}$ & - \\
\hline $\begin{array}{l}\text { Proximal to intron } 62 \\
\text { Distal to intron } 62\end{array}$ & $\begin{array}{l}\text { Dp427, Dp260, Dp140, Dp116 } \\
\text { +Dp71, Dp40 }\end{array}$ & $\begin{array}{r}35 \\
2\end{array}$ & $\begin{array}{l}89.66(16.97) \\
51.00(9.90)^{c}\end{array}$ & - \\
\hline
\end{tabular}

$n$ : number of patients; FSIQ: full scale intelligence quotient; SD: standard deviation; Dp140: Dp140utr+Dp140pc; Dp140utr: 5' untranslated region of Dp140; Dp140pc: promoter and protein coding region of Dp140; +: additionally affected dystrophin isoforms; -: only few observation in one group, statistical test was not reliable.

${ }^{\text {a }} p<0.01$ statistical significance (Bonferroni correction 0.05/3).

${ }^{\mathrm{b}}$ Three patients with FSIQ of 55, 44 and 58.

${ }^{c}$ Two patients with FSIQ of 44 and 58. 


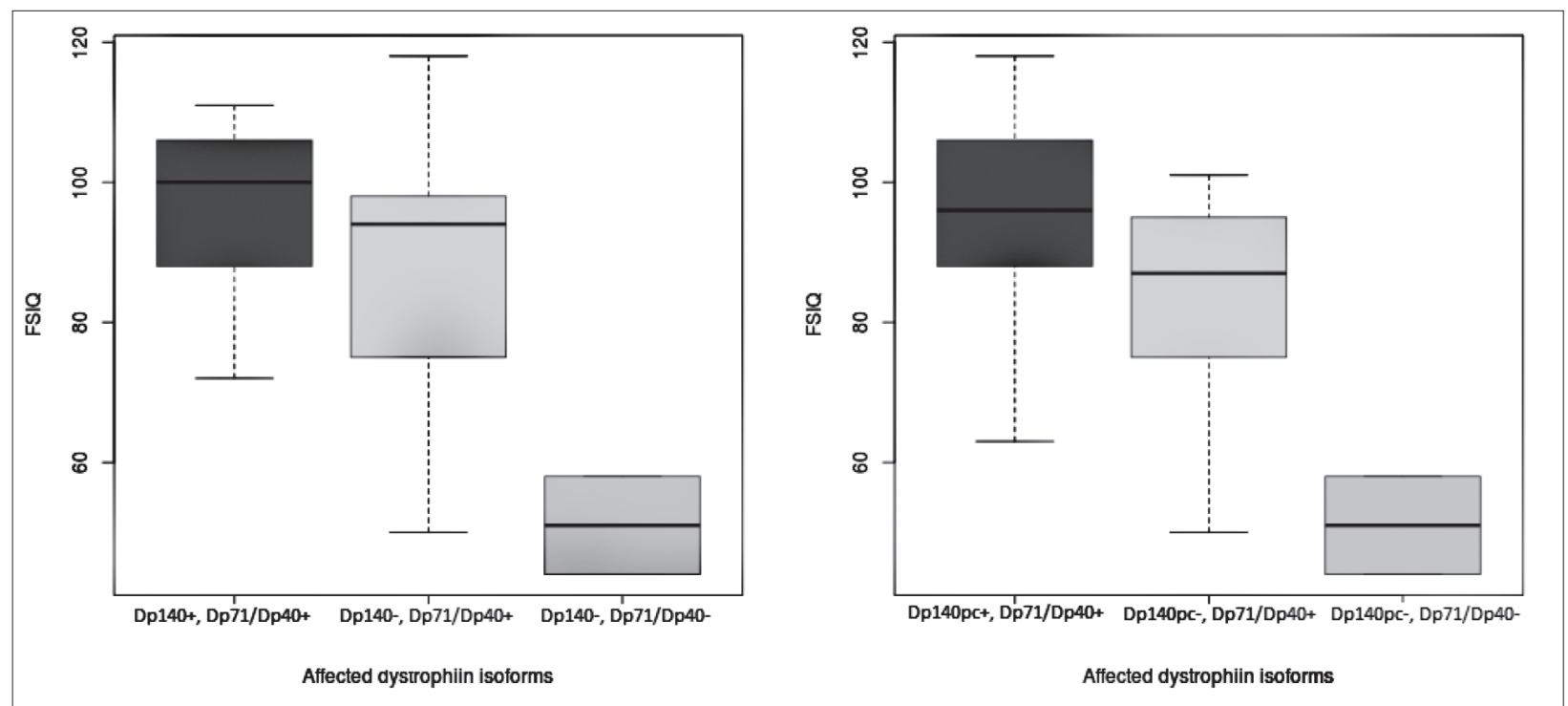

Figure 1. Distribution of FSIQ with respect to the preservation or loss of Dp140 and Dp71/Dp40 indicates that cognitive impairment in DMD patients is associated with the cumulative loss of dystrophin isoforms. A greater proportion of variability on FSIQ was explained after clustering mutations within the Dp140utr together with mutations affecting expression of Dp427 and Dp260 isofroms, but no expression of the Dp140 isoform. FSIQ: full scale intelligence quotient; +/-: preservation/absence of appropriate dystrophin isoform; Dp140-Dp140utr+Dp140pc: Dp140utr: 5' untranslated region of Dp140; Dp140pc: promoter and protein coding region of Dp140.

Dp140utr, while slightly delayed psychomotor development $(\mathrm{DQ}=82)$ was reported for the other boy (ID 40) whose mutation affected expression of all dystrophin isoforms.

Moreover, for two DMD patients with difficulties in a test situation, SQ was estimated. One patient (ID 38), with autistic like-behavior and undeveloped speech, had SQ 41, while the other (ID 41) had SQ 58. The patient with autistic like-behavior had a deletion that altered expression of Dp427 only, while duplication/triplication in the distal part of the $D M D$ gene caused loss of all dystrophin isoforms in the other patient. Cognitive assessment for one sibling pair showed intellectual impairment in one sibling (ID 37), while slightly delayed psychomotor development was noted in the other (ID 40).

\section{DISCUSSION}

The aim of this study was to determine the frequency of intellectual impairment and relationship between intelligence level and dystrophin mutations in the Serbian group of patients with DMD. Consistent with previously published studies, general intellectual level in our study group was statistically significantly different from normative values [27].
The FSIQ was reduced for almost 1 SD (15 FSIQ points) from population average, which is in agreement with the results from other DMD cohorts [2830], or DMD plus intermediate MD cohorts [31]. Seven boys in our sample (18.92\%) had intellectual impairment with FSIQ $<70$, which is slightly lower but still in agreement with the results reported in other studies (19-35\%) [2,3,28-30].

The majority of patients in our study had confirmed causal deletion in the $D M D$ gene, so it was difficult to analyze the effect of mutation type on intellectual level. Even though only four patients had duplication in the $D M D$ gene, three of them (IDs 3, 6 and 7) had normal intelligence ability, while for one patient (ID 41), the estimated SQ, which highly correlates with FSIQ, was low. The patients with normal intellectual ability had a duplication in the proximal part of the $D M D$ gene that affected expression of Dp427, while the patient with low SQ had a duplication/triplication that altered the expression of all dystrophin isoforms. Our study did not include patients with DMD clinical presentation without deletion or duplication and with possible point mutations. However, Taylor et al. [14] published a study in which there was no significant correlation between mutation type and FSIQ. 
Despite the fact that some of the previously published studies found association between the structural location of mutations and FSIQ [22,23,28], we were not able to replicate this association in our study when boundaries for proximal and distal mutations were set at exon 30 and exon 45. However, association between the intellectual ability of DMD patients and mutation location in regard to their functional consequence, loss of expression of different dystrophin isoforms [14], was confirmed with our results.

The loss of the Dp427 isoform is a common feature among all DMD patients, which may result in cognitive impairment. Dp427 is expressed in the neocortex, cerebellum and amygdala $[6,32,33]$, where it plays not only a structural role in central synapses but likely regulates $\mathrm{GABA}_{\mathrm{A}}$ receptor clustering at inhibitory synapses [9]. In eight patients whose mutations abolished the expression of Dp427, two boys (IDs 1 and 4) (25.0\%) had borderline FSIQ, while none had an intellectual impairment (FSIQ $<70)$. However, the mutations affecting the expression of Dp140 and Dp71/Dp40, in addition to Dp427, have been associated with higher frequency and severe cognitive impairment in our DMD patients, suggesting the effect of cumulative loss of dystrophin isoforms and the important role of Dp140 and Dp71/Dp40 on intellectual ability. This finding is in agreement with the results of previously published studies [14,15,34-36].

The role of the Dp140 isoform on intellectual functioning was recognized by Felisariet et al. [37], who described the association between the mutations affecting the expression of Dp140 and intellectual ability in DMD patients, has been replicated in other studies $[14,28,29]$. Dp140 is detected throughout the brain (cerebral cortex, cerebellum, hippocampus, brain stem and olfactory bulb) and in the spinal cord [11], but its function is still unclear. Our results suggest statistically significantly lower FSIQ in patients with altered Dp140, Dp116, Dp71 and Dp40 compared to patients with the mutations affecting only the expression of Dp427 and Dp260. However, a statistically significant difference has been obtained after clustering mutations in the Dp140utr region together with the mutations affecting the expression of Dp427 and Dp260. Additionally, statistically significant difference in the mean FSIQ was obtained when patients with the mutation affecting Dp140 were classified into groups with the mutation localization in 5'UTR (Dp140utr) or in promoter and protein-coding region
(Dp140pc). These results underline the importance of assuming that the expression of the Dp140 isoform is not mainly affected by mutations located in its long 5'UTR, which includes frequently deleted exons 4550 . All the same, in our group of patients, three boys (IDs 14, 19 and 21) with mutations in the Dp140utr coding region were intellectually impaired, suggesting that some regulatory elements in the 5'UTR might be affected, influencing the alteration in the expression of Dp140. Our results are in agreement with a previously published study suggesting that mutations in the Dp140utr have a lesser effect on FSIQ when compared to the mutations affecting Dp140pc [14].

The Dp71 isoform is a major product of the $D M D$ gene in the brain. It has been confirmed that Dp71 is abundant in the fetal as well as adult brain, particularly in the cerebral cortex and hippocampus [38]. Although the function of this isoform remains unknown, it has been reported that Dp71 has a role in the stabilization and/or formation of the synaptic membrane [39]. The dysfunctions of proteins involved in the regulation of synaptic structure and function influence neuronal connectivity and the ability of the brain to process information, and may be related to the cognitive impairment [36]. Additionally, it was shown that Dp71 has a regulatory role in excitatory synapse organization and function, by clustering glutamate receptors and organizing signaling in postsynaptic densities [15]. The systematic occurrence of mild-to-severe mental retardation was noticed in more than 50 patients with dystrophinopathies and the mutation located in the Dp71 [15]. Although the number of patients with altered Dp71 in our study was small $(n=2)$, which is in accordance with the low frequency of deletions and duplications in the most distal part of the $D M D$ gene, both patients (IDs 36 and 37) were intellectually disabled, whereas one (ID 36) had the lowest FSIQ (44) within the entire study group. The mutations affecting the expression of Dp71 also effect the expression of all dystrophin isoforms, supporting the importance of cumulative loss of dystrophin isoforms apart from the loss of Dp71 only, reported in this and other studies $[14,15,34]$.

Until recently, the role of the shortest isoform Dp40 in the brain was unknown. A finding that Dp40 is enriched in the synaptic vesicle fraction where it assembles a group of presynaptic proteins involved in the exocytosis of synaptic vesicles, indicates that 
Dp40 might have an important role in presynaptic function [16]. Even though other studies did not analyze the effect of Dp40, the patients with intellec-tual impairment had mutations that influenced the expression of both Dp71 and Dp40 [15,16,34], implying that $\mathrm{Dp} 40$ might have a function relevant to cognitive processes.

Interestingly, one pair of siblings who were observed in our study, had the mutation that affects Dp71, but the use of different psychological instruments made it difficult to correlate genetic data with cognitive ability assessment and to analyze variation in DMD expression between siblings (IDs 37 and 40) with the same mutations. Unlike the FSIQ, the DQ is a ratio reflecting the child's overall development without precisely defined correlation with FSIQ in later life. Still, the infant who scores low often turns out to be intellectually disabled [40].

In addition, the association of DMD with neuropsychiatric disorders has also been recognized. Wu et al. [41] published a study in which they confirmed a previously unrecognized relation between DMD and an autistic spectrum disorder. Therefore, it is not surprising that one boy (ID 38) with autism likebehavior, qualitatively different from the behavior of DMD boys with mental retardation, was described in our sample. His SQ, which was estimated to be 41 , indicates below average achievement and impairment in adaptability, including communication, daily living, and socialization. The mutation in this boy affected only the expression of Dp427.

The limitations of our study were the retrospective design and limited sample size, but since psychological testing is a standard procedure in the care of DMD patients at the Clinic for Neurology and Psychiatry for Children and Youth, Belgrade, Serbia, we enabled an unbiased selection of recruited patients, overcoming selection issues discussed in other studies [2]. The non longitudinal design of our study excluded the possibilities of defining subtypes within DMD [23] and to define the clinical severity of two very young DMD patients (IDs 2 and 38) with inframe mutations. In general, in-frame mutations are associated with milder form of dystrophinopathy, but exceptions to the reading frame hypothesis exist [42].

In summary, the classification of the mutations based on their functional consequence on dystrophin isoforms, with the assumption that the expression of Dp140 is not mainly affected by the mutations in its 5'UTR, explained the genetic influence on variability of FSIQ with the effect of cumulative loss of dystrophin isoforms, suggesting an important role of Dp140, Dp71 and Dp40 isoforms on intellectual ability. Defining the functional loss of dystro-phin isoforms allows the recognition of the subgroup of DMD boys with greater risk for cognitive problems. Early interventions and the support in cognitive, emotional and behavioral development could be very useful and more effective than interventions in the older period of childhood or adolescence.

Declaration of Interest. This study was supported by a grant from the Ministry of Education, Science and Technological Development, Republic of Serbia (project Nos. 173016 and 17 508). The authors report no conflicts of interest. The authors alone are responsible for the content and writing of this article.

\section{ACKNOWLEDGMENTS}

We thank the patients and their families for facilitating this research. VMR and DSP designed the study; VMR, JM, AK and ST recruited the subjects, gathered the clinical data and contributed DNA samples; JP, IN, NM, DSP and SR performed and analyzed the genetic tests; VL and GM performed the psychological tests; DV performed statistical analyses; VMR, DV, JP and DSP wrote the manuscript.

\section{REFERENCES}

1. Emery A, Muntoni F. Duchenne muscular dystrophy. 3rd ed. New York, NY: Oxford University Press, 2003.

2. Cotton S, Voudouris NJ, Greenwood KM. Intelligence and Duchenne muscular dystrophy: Full-scale, verbal, and performance intelligence quotients. Dev Med Child Neurol. 2001; 43(7): 497-501.

3. Cotton S, Voudouris N, Douglas J. Key findings from a meta-analytical study on intellectual functions in Duchenne muscular dystrophy. J Intell Disabil Res. 2000; 44(3-4): 248-248.

4. Koenig M, Hoffman EP, Bertelson CJ, Monaco AP, Feener C, Kunkel LM. Complete cloning of the Duchenne muscular dystrophy (DMD) cDNA and preliminary genomic organization of 
the DMD gene in normal and affected individuals. Cell. 1987; 50(3): 509-517.

5. Monaco AP, Bertelson CJ, Liechti-Gallati S, Moser H, Kunkel LM. An explanation for the phenotypic differences between patients bearing partial deletions of the DMD locus. Genomics. 1988; 2(1): 90-95.

6. Lidov HG, Byers TJ, Watkins SC, Kunkel LM. Localization of dystrophin to postsynaptic regions of central nervous system cortical neurons. Nature. 1990; 348(6303): 725-728.

7. Tokarz SA, Duncan NM, Rash SM, Sadeghi A, Dewan AK, Pillers DA. Redefinition of dystrophin isoform distribution in mouse tissue by RTPCR implies role in nonmuscle manifestations of duchenne muscular dystrophy. Mol Genet Metab. 1998; 65(4): 272-281.

8. Anderson JL, Head SI, Rae C, Morley JW. Brain function in Duchenne muscular dystrophy. Brain. 2002; 125(1): 4-13.

9. Perronnet C, Vaillend C. Dystrophins, utrophins, and associated scaffolding complexes: Role in mammalian brain and implications for therapeutic strategies. J Biomed Biotechnol. 2010; 2010: 849426. doi: 10.1155/2010/849426.

10. Pillers DA, Bulman DE, Weleber RG, Sigesmund DA, Musarella MA, Powell BR, et al. Dystrophin expression in the human retina is required for normal function as defined by electroretinography. Nat Genet. 1993; 4(1): 82-86.

11. Lidov HG, Selig S, Kunkel LM. Dp140: A novel $140 \mathrm{kDa}$ CNS transcript from the dystrophin locus. Hum Mol Genet. 1995; 4(3): 329-335.

12. Byers TJ, Lidov HG, Kunkel LM. An alternative dystrophin transcript specific to peripheral nerve. Nat Genet. 1993; 4(1): 77-81.

13. Bar S, Barnea E, Levy Z, Neuman S, Yaffe D, Nudel U. A novel product of the Duchenne muscular dystrophy gene which greatly differs from the known isoforms in its structure and tissue distribution. Biochem J. 1990; 272(2): 557-560.

14. Taylor PJ, Betts GA, Maroulis S, Gilissen C, Pedersen RL, Mowat DR, et al. Dystrophin gene mutation location and the risk of cognitive impairment in Duchenne muscular dystrophy. PLoS One. 2010; 5(1): e8803. doi: 10.1371/ journal.pone.0008803.
15. Daoud F, Angeard N, Demerre B, Martie I, Benyaou R, Leturcq F, et al. Analysis of Dp71 contribution in the severity of mental retardation through comparison of Duchenne and Becker patients differing by mutation consequences on Dp71 expression. Hum Mol Genet. 2009; 18(20): 3779-3794.

16. Tozawa T, Itoh K, Yaoi T, Tando S, Umekage M, Dai H, et al. The shortest isoform of dystrophin (Dp40) interacts with a group of presynaptic proteins to form a presumptive novel complex in the mouse brain. Mol Neu-robiol. 2012; 45(2): 287-297.

17. Schouten JP, McElgunn CJ, Waaijer R, Zwijnenburg D, Diepvens F, Pals G. Relative quantification of 40 nucleic acid sequences by multiplex ligationdependent probe amplification. Nucleic Acids Res. 2002; 30(12): e57. doi: 10.1093/nar/gnf056.

18. Chamberlain JS, Gibbs RA, Ranier JE, Nguyen PN, Caskey CT. Deletion screening of the Duchenne muscular dystrophy locus via multiplex DNA amplification. Nucleic Acids Res. 1988; 16(23): 11141-11156.

19. Beggs AH, Koenig M, Boyce FM, Kunkel LM. Detection of $98 \%$ of DMD/BMD gene deletions by polymerase chain reaction. Hum Genet. 1990; 86(1): 45-48.

20. Kunkel LM, Snyder JR, Beggs AH, Boyce FM, Feener CA. Searching for dystrophin gene deletions in patients with atypical presentations. In: Lindsten J, Petterson U, Eds. Etiology of Human Diseases at the DNA Level. New York, NY: Raven Press. 1991: 51-61.

21. Den Dunnen JT, Antonarakis SE. Mutation nomenclature extensions and suggestions to describe complex mutations: A discussion. Hum Mutat. 2000; 15(1): 7-12.

22. Bushby KM, Appleton R, Anderson LV, Welch JL, Kelly P, Gardner-Medwin D. Deletion status and intellectual impairment in Duchenne muscular dystrophy. Dev Med Child Neurol. 1995; 37(3): 260-269.

23. Desguerre I, Christov C, Mayer M, Zeller R, Becane HM, Bastuji-Garin S, et al. Clinical heterogeneity of Duchenne Muscular Dystrophy (DMD): Definition of sub-phenotypes and predictive criteria by long-term follow-up. PLoS One. 2009; 4(2): e4347. doi: 10.1371/ ournal.pone.0004347. 
24. Wechsler D. Manual for the Wechsler Intelligence Scale for Children, 3rd ed. New York, NY: Psychological Corporation, 1991.

25. Brunet O, Lezine PUF. Le Developpement Psychologique del al Premiere Enfance. Issy-lesMoulineaux. France: Editions Scientifiques et Psychotechniques, 1951.

26. Doll EA. Vineland Social Maturity Scale. Oxford, UK: Educational Test Bureau, 1947.

27. Hinton VJ, Fee RJ, Goldstein EM, De Vivo DC. Verbal and memory skills in males with Duchenne muscular dystrophy. Dev Med Child Neurol. 2007; 49(2): 123-128.

28. D'Angelo MG, Lorusso ML, Civati F, Comi GP, Magri F, Del Bo R, et al. Neurocognitive profiles in Duchenne muscular dystrophy and gene mutation site. Pediatr Neurol. 2011; 45(5): 292-299.

29. Wingeier K, Giger E, Strozzi S, Kreis R, Joncourt F, Conrad B, et al. Neuropsychological impairments and the impact of dystrophin mutations on general cognitive functioning of patients with Duchenne muscular dystrophy. J Clin Neurosci. 2011; 18(1): 90-95.

30. Lorusso ML, Civati F, Molteni M, Turconi AC, Bresolin N, D'Angelo MG. Specific profiles of neurocognitive and reading functions in a sample of 42 Italian boys with Duchenne Muscular Dystrophy. Child Neuropsychol. 2013; 19(4): 350-369.

31. Chamova T, Guergueltcheva V, Raycheva M, Todorov T, Genova F, Bichev S, et al. Association between loss of Dp140 and cognitive impairment in Du-henne and Becker dystrophies. Balkan J Med Genet. 2013; 16(1): 21-30.

32. Knuesel I, Bornhauser BC, Zuellig RA, Heller F, Schaub MC, Fritschy JM. Differential expression of utrophin and dystrophin in CNS neurons: An in situ hybridization and immunohistochemical study. J Comp Neurol. 2000; 422(4): 594-611.

33. Sekiguchi M, Zushida K, Yoshida M, Maekawa M, Kamichi S, Yoshida M, et al. A deficit of brain dystrophin impairs specific amygdala GABAergic transmission and enhances defensive behaviour in mice. Brain. 2009; 132(1): 124-135.

34. Moizard MP, Toutain A, Fournier D, Berret F, Raynaud M, Billard C, et al. Severe cognitive impairment in DMD: Obvious clinical indication for Dp71 isoform point mutation screening. Eur J Hum Genet. 2000; 8(7): 552-556.

35. Moizard MP, Billard C, Toutain A, Berret F, Marmin N, Moraine C. Are Dp71 and Dp140 brain dystrophin isoforms related to cognitive impairment in Duchenne muscular dystrophy? Am J Med Genet. 1998; 80(1): 32-41.

36. Chelly J, Khelfaoui M, Francis F, Cherif B, Bienvenu T. Genetics and pathophysiology of mental retardation. Eur J Hum Genet. 2006; 14(6): 701-713.

37. Felisari G, Martinelli Boneschi F, Bardoni A, Sironi M, Comi GP, Robotti M, et al. Loss of Dp140 dystrophin isoform and intellectual impairment in Duchenne dystrophy. Neurology. 2000; 55(4): 559-564.

38. Haenggi T, Soontornmalai A, Schaub MC, Fritschy JM. The role of utrophin and Dp71 for assembly of different dystrophin-associated protein complexes (DPCs) in the choroid plexus and microvasculature of the brain. Neuroscience. 2004; 129(2): 403-413.

39. Jung D, Yang B, Meyer J, Chamberlain JS, Campbell KP. Identification and characterization of the dystrophin anchoring site on $\beta$-dystroglycan. J Biol Chem. 1995; 270(45): 27305-27310.

40. Sigelman C, Rider EA. Life-Span Human Development, 7th ed. Belmont, CA: Wadsworth Cengage Learning, 2012.

41. Wu JY, Kuban KC, Allred E, Shapiro F, Darras BT. Association of Duchenne muscular dystrophy with autism spectrum disorder. J Child Neurol. 2005; 20(10): 790-795.

42. Ferlini A, Neri M, Gualandi F. The medical genetics of dystrophinopathies: Molecular genetic diagnosis and its impact on clinical practice. Neuromusc Disord. 2013; 23(1): 4-14. 